\title{
Recent Advances in Conformal Antennas and its Design Issues
}

\author{
K. Sumalatha, Ch. R. Phani Kumar
}

\begin{abstract}
With the rapid progress of the use of miniature sized antennas, wireless communication technology has quickly risen. Not only have the size of the antenna but also its cost, efficiency and ease of assembly all been taken into account during the antenna design. Conformal antennas are suggested to satisfy all these requirements. Conformal antennas have broad commercial, defense and several civil services applications. In aircraft and boats it is the most important and necessary time. Patch antennas are often regarded the best choice for such conformal antennas. In this paper, we address the conformal transmitters with different substrates used for antenna design. With this article, we create a modeling of conformal antennas with latest developments by examining early events.
\end{abstract}

Index Terms: Conformal antennas, wireless communication technology, Patch antennas, commercial, defense.

\section{INTRODUCTION}

The conformal receiver is a radiation frame as per IEC (International Electro-technical Commission) by its electromagnetic characteristics, the form of which is not settled $[1,2]$. By the frame surface with progress in new techniques, and development the device setup must be accepted. An ordinary radiating scheme refers in free space to a scheme that runs as the interface between the generator and the receiver. In order to improve general system efficiency, the features can be changed. This antenna can be implemented on a few aerodynamic devices. They have group broadband, miniaturization and multiband features, in addition to conformal nature.

Conformal antenna has a position in the category of the phased array. They contain a range of small flat antennas such as pads and dipoles. Conformal antennas are used in countless applications when arranging notable antennas. Antenna installation on circular panels for wider inclusion [3, 4], in an airplane or vehicle [5-7], special composites for load-bearing reasons for the installation of transmitters [8], for wearable mobile networks devices as fabric antennas [9-11] may be included. The antenna devices must operate on a variety of such applications with increasingly adaptive beam-forming capacity, radiation effectiveness, structural strength and cost-effective RF equipment. In addition, countless new RF circuit study regions [12], E-textile conductors [13] and metalized fibers [14] have been generated for the complicated concept of these applications in complicated radiation environments. These attempts have generated remarkable features and numerous of the issues

Revised Manuscript Received on July 22, 2019

K. Sumalatha, Research Scholar GITAM University, and Working as Associate Professor, Dept. of ECE, Malla Reddy Engineering College for Women, Hyderabad, Telangana, India.

Dr. Ch. R. Phani Kumar, Assistant Professor, GITAM University, Vizag, Andhra Pradesh, India

arising with the physical application of conformal antennas. Due to unwanted surface deformations, the radiation features of these conformal amplifiers can be significantly degraded [15]. Due to its elevated profitability, beam shaping and beam steering, the antenna array has taken increasing regard since late. The primary aim is to find the appropriate excitation stage and amplitude to synthesize the optimal radiation model in antenna array model synthesis. Different approaches including optimization algorithms and traditional mathematical structures have been implemented to address complicated antenna model synthesis [16, 17]. The traditional mathematical scheme is mostly used for linear homogeneous arrays. The conformal arrays of components broadly guide their radiation rays to different coils in bent surfaces and not all components make a similar contribution to the required model. In model synthesis, this feature results in model synthesis difficulties.

In the past, study has shown that with adequate passive and active mechanical damping, mechanical steering, stage compensation and tension amplitudes and actuators, radiation patterns may be increased as conformal antenna structure modifications [18]. The focus of this study is on the present ground change, polarization and model synthesis practices, methodologies, instruments and frames of conformal antennas. The main contributions and organization of this paper are summarized as follows: In section 2 we describe background details of the various modeling of conformal antenna. The section 3 proposed work. Finally, in section 4 we concluded the paper.

\section{BACKGROUND WORKS}

Considering the recent advancements in the wireless technology, there are numerous approaches to design conformal antenna. This section glances at different existing techniques. The surveys were selected to incorporate the current procedures and methods applied to different information for design of conformal antenna. Examined techniques and methods are within the past recent years that fall in categories of changing surfaces, polarization and pattern synthesis techniques. Fig. 1 shows the modeling of conformal antenna with various methods.

A. Modeling of Conformal Antennas for Changing Surfaces

In [19], the authors displayed a phased-array experiment phase to focus the capacity of conformal antennas self-adapting capabilities. The writers have intended a four-port receiver $(2.45 \mathrm{GHz})$ with power supply regulated attenuators and phase shifters, in addition to four separate colored micro-stream panel antennas linked to a conformal ground. 


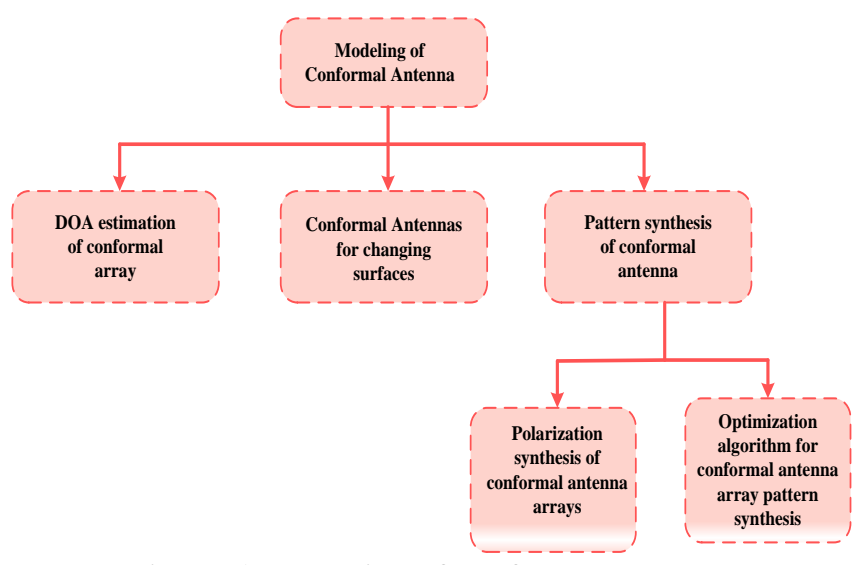

Figure 1. Modeling of conformal antenna

With a flexible SMA Cable every antenna was associated with the comparing receiver port. The developing of the conformal array with suitable phase compensation brought several changes by making proper coupling of distortion pattern of antenna. To build up another self-adapting flexible micro strip with an integration with the system this pattern recovery data was utilized. To measure the surface deformation of the antenna and to control the phase shifters the sensor circuit was exploited.

In [20], the authors exhibited a millimeter-wave shaped-beam substrate integrated conformal array antenna. In the wake of examining the conformal shape impact on the characteristics of a radiating slot and a Substrate Integrated Waveguide (SIW), on a cylindrical surface the array mounted with a radius of $20 \mathrm{~mm}$ is synthesized at the center frequency (35 GHz). In a solitary dielectric substrate all components, including a phase compensated network, $8 \times 8$ slot array and 1-to- 8 divider were created together. At the beam direction the cross polarization was lower than $-41.7 \mathrm{db}$. In millimeter-wave frequency band, the presented SIW conspire had the capacity to illuminate the trouble of reconciliation between a feed network and conformal array elements. From the feed network, while maintaining a strategic distance from element-to-element parasitic cross-coupling and spillage in radiation.

The writers investigated the self-adaptation conformal antennas for altering spherical plates in [21]. In specific, the hypothesis was developed about the connection between conformal array element spacing, necessary phase compensation, and spherical surface radius. For theoretical authorization, the radiators are used at $2.47 \mathrm{HGz}$ with separate micro strip antennas. The detecting system was used to quantify the radius of curvature of the spherical surface. These information are used autonomously to retrieve the radiation pattern of the set on the different spherical bodies at $2.47 \mathrm{GHz}$ depending on previous theoretical advances.

To changing conformal surfaces the authors in [22] was created another $1 \times 4$ frequency reconfigurable self-adapting conformal antenna array. The displayed conformal array comprises of four reconfigurable elements of micro-strip patch antenna. To quantify the voltage controlled phase shifters and the curvature of conformal surface a reconfigurable sensor circuit was utilized. By the reconfigurable sensing circuit these phase shifters were controlled. The phase compensation was actualized to autonomously recuperate the pattern in the two bands of the reconfigurable; the array was appended upon shape change.

Authors showed a conformal wide-band SIW H-plane horn antenna installed on a significant transmitting cylinder [23].
When installed on a cylinder of moving radius, the effect of a curved low-profile SIW H-plane horn was checked. The two air slots along the ends of the antenna flare were cut to recompense for the H-plane horn antenna's VSWR. The match between the horn antenna and the feed line was improved with the use of amplifiers, while the operating frequency bandwidth was expanded. In their work from $6.1 \mathrm{GHZ}$ to $19 \mathrm{GHZ}$ the VSWR measured of the fabricated antenna was below 2.5 over the same frequency range the antenna likewise shows stable radiation.

\section{B. DOA Estimation of Conformal Array}

For DOA estimation of conformal array, several algorithms have already presented [24-28].

In a Massive Multiple-Input-Multiple-Output (MIMO) framework under obscure mutual coupling, a novel 2D DOA estimation approach dependent on the tensor method was presented by authors in [24] for a conformal array. The declaration of received signal was defined with the Khatri Rao (KR) product by setting sensors uniformly on the cylindrical surface. In light of the conformal array an obscure mutual coupling auto-suppression technique was researched. To use the multidimensional data of the received information dependent on the conformal array signal model a third-order tensor was constructed. By HOSVD (Higher-Order Singular Value Decomposition) moreover the signal subspace was given. At long last, by conventional subspace-based algorithms the DOAs were estimated.

Based on conformal array a 2-D DOA estimation algorithm for coherently distributed (CD) sources was presented authors in [25]. In view of GSVS (Generalized Steering Vectors) three rational invariance relationships were built. For estimating three rational invariance matrices then the PM (Propagator Method) was utilized. At last, from the Eigen values of three rational invariance matrices the 2-D DOA of CD sources were gotten. The presented algorithm have low computational complexity without Eigen decomposition of sampling covariance matrix and searching of spectrum peaking and estimation.

A novel high accuracy 2D-DOA estimation algorithm for the conformal array was introduced by authors in [26]. By means of PARAFAC (Parallel Factor Analysis) theory a 2D-DOA estimation of the cylindrical conformal exhibit was practiced. Due to the polarization diversity of the fluctuating curvature the customary DOA estimation algorithm can't be utilized on conformal array. In the covariance domain the algorithm shapes a PARAFAC model of the covariance matrices to stay away from the issue of parameter pairing. To other conformal array structures and non-uniform noise scenario the presented algorithm was likewise summed up. To confirm the execution of the presented algorithm the simulation results with the cylindrical conformal array were introduced and the CRB (Cramer-Rao Bound) was derived.

A conformal cylindrical micro strip antenna array was planned and explored by the authors in [27]. To investigate the superior of 2-D DOA estimation, A NC-MUSIC (non-circular music) algorithm with a conformal cylindrical array was incorporated. For every element in the antenna array the electric fields were dissected and considered the impacts of it on NC-MUSIC algorithm. Their reenactment results demonstrates that the presented technique have expanded the most extreme estimation number of NC-MUSIC algorithm and 
have higher resolution than the standard music algorithm. In ITS (Intelligent Transportation Systems) with conformal antenna, a mutual coupling coefficient and a novel DOA estimation algorithm was presented by the authors in [28]. Alongside instrumental element technique the impact of mutual coupling was wiped out by developing the special MCM (mutual coupling matrix). In view of PARAFAC theory, the DOA of incident signals was estimated. In cumulate domain utilizing covariance matrices the PARAFAC demonstrate was developed. In light of the matrix transformation between the steering vector and MCM the mutual coupling coefficients were evaluated.

\section{Pattern Synthesis of Conformal Antenna}

In this section the pattern synthesis of conformal antenna based on optimization algorithms and polarization with different approaches is discussed.

\section{Optimization Algorithm for Conformal Antenna Array Pattern Synthesis}

The writers provided a hybrid GA-PSO algorithm (Genetic Algorithm and Particle Swarm Optimization) for model synthesis of conformal antenna arrays [29]. When linked to antenna model syntheses using the two strategies benefits, the presented technique has elevated convergence precision and quick convergence rate. A few median sample features and optimization cases of a linear array model synthesis demonstrated the hybrid optimization algorithm implementation. To finally display the submitted algorithm, a cylindrical conformal micro strip antenna array was examined as a basis for earth synthesis. The presented technique has been accurate and convincing for the conformal antenna array model synthesis. In view of necessities on both excitation DRR (Dynamic Range Ratio) and polarization, the writers in [30] presented an iterative method for the 3D synthesis of a conformal antenna array's electrical far-field model. In a specified angular region, the presented calculation allows a flexible polarization and DRR control. The range of excitations needs small CPU time. A modified algorithm adjustment was also developed to optimize the polarization condition by phase-only command. For joint polarization and model extraction, numerical results of conformal models with lessened DRR were presented to verify the usefulness of the methods shown.

The authors in [31] generally tend to conform array synthesis as a multi-objective optimization issue. With a restriction on basic beam orientation, XPL (cross-polarization level) and SLL (side lobe level) were synchronously decreased. To optimize model, a WARP (weighted alternative inverse projection) and 2LB-MOPSO (Two Local Best Multi-Objective Particle Swarm Optimization) hybrid was provided. In their first studies, the WARP technique discovers a viable, mild alternative. At that stage, 2LB-MOPSO method begins, integrating the WARP alternative with an inherent demographic and punishment capacity for managing restriction. In the fundamental 2LB-MOPSO population, including the WARP consequence, local extreme traps are evaded, less awareness and greater resistance to penalty features. After appropriate iterations, the submitted method provides superior SLL and XPL related to the fast stagnating WARP technique.

In [32], for conformal antenna systems, a fast approach was provided that empowers cross-polar pattern synthesis, 3D co-polar, concurrently reducing the array excitations DRR. By introducing in specific two additional stage models, one for the co-polar model and the other for the cross-polar pattern, the energy transmission problem was reduced to field synthesis version. The problem was then iteratively resolved on the array excitations and two additional stage models. For the particular case, the altered version of the technique was introduced where DRR decrease is not required, yet the computational time was also reduced. The authors implemented a method for encouraging conformal array element construction [33]. Using a model-based parameter assessment method (MBPE) to interpolate element models at selected locations, the method shown demonstrates images of an item at a subjective panel place. Interpolation approach has two phases. It interpolates the Fourier number on the specified pattern element locations, then unites the element to the IFFT / IDFT (Inverse Fast Fourier transforms the IDFT) algorithm. PSO method optimized element design.

An improved NSGA-II models and VSIE (volume-surface differential equation) were produced and studied by the writers in [34]. With the VSIE algorithm, port characteristics and antenna element models with shared bonding were re-enacted. By their distinctive impedances labeled active element models, all element models were finished and for correlation evaluated in a microwave room. Between evaluated and repeated results, their results show a good game. With distinct weights of all these active element models, the conformal antenna grid model was a superposition. In any event, the setup of items comprising side lobe concentrations, beam orientation and 3-db beam width was regarded. The INSGA-II algorithm was used for a multi-object optimization.

In model synthesis, the writers in [35] provided a hybrid IWO (invasive plant optimization) and PSO (particle swarm optimization) algorithm. The submitted algorithm was regarded a cylindrical conformal micro strip array. Using the hybrid IWO / PSO circuit, the amplitude and stage of each excited voltage were optimized and the active element model of each component in the conformal matrix was removed. Exploratory results show that while barring their distinct deficiencies, the submitted hybrid algorithm retains the specific benefits of IWO and PSO calculations when compared and conventional PSO and IWO models. Moreover, the submitted hybrid algorithm was very accurate in achieving worldwide optimality. The writers provided a convex optimization based complete polar metric amount and pattern synthesis method for a conformal array [36]. To show the conformal array response, the approach gets co-polarization, co-polarization and multiple segregation from display calibration readings. Plan the convex optimization problems as pattern synthesis of polarization ratio and difference at that stage. The method was linked to a recreated conformal spherical conformal array of 176 antenna components and the small cross-polarization frequency distinction and sum models were described.

\section{Polarization Synthesis of Conformal Antenna Arrays:}

The authors in [37] related a convex TO conformal antenna array theory to synthesize dual polarization models of accurate polar metric radar identification. The developers discovered optimization in convex shape and the parameters guarantee method was shown. To demonstrate the ability of the mentioned strategy, two array configurations were introduced: cylindrical and spherical arrays of merged

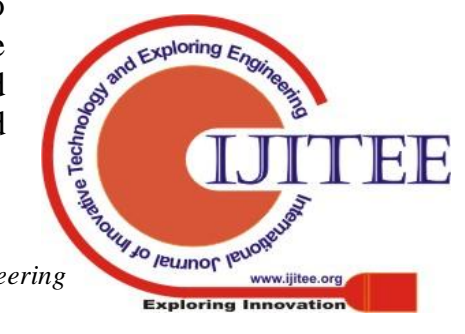


dipoles, and double-polarized locations. The authors shifted towards composing a rare conformal stage set with parts on a truncated cone [38]. The antenna should be a fully electronic receiver of an aerial traffic control system, to cover a necessary angular region, it must concurrently produce different receiving waves along its lift. To characterize the complex excitation variety for each individual beam and array component sites, Bayesian Compressive Sensing (MTBCS) methods completed the synthesis issue.

\section{METHOD \& METHODOLOGY}

This U-slot patch antenna is then mounted on a cylindrical structure with a radius $r$ and an arc angle $\alpha$, as shown in Figure. 2, where the antenna is bent along its $H$-plane, i.e., the patch width. The planar and conformal wideband antennas are numerically analyzed using a finite-element based full-wave electromagnetic solver, ANSYS HFSS v.16 [12].

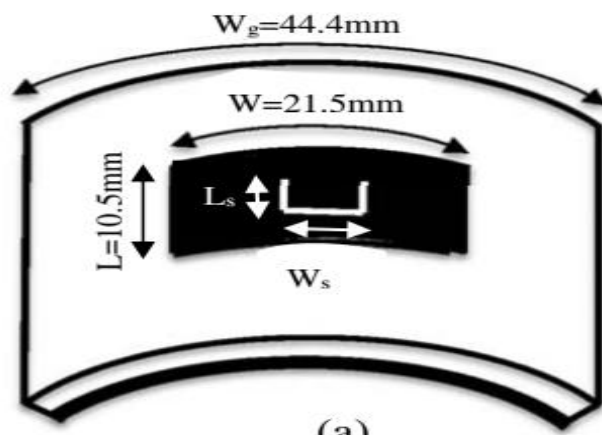

(a)

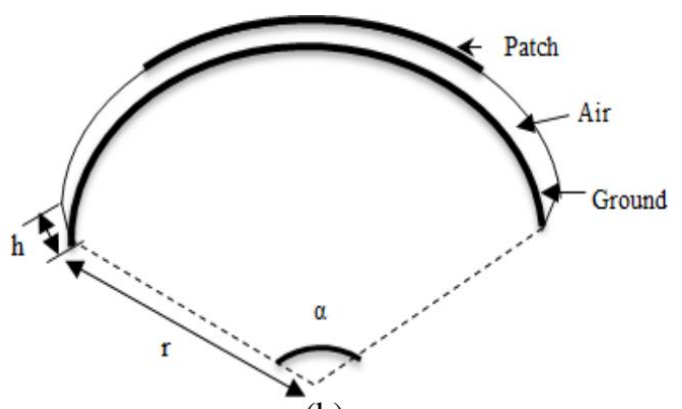

(b)

Figure 2. (a) Top- and (b) side-view of the conformal wideband antenna, placed on a cylindrical mounting structure and bent along the $H$-plane with $h=3.175 \mathrm{~mm}$ [40]

\section{RESULTS \& DISCUSSION}

The scattering parameters, $|S 11|$, for the planar and conformal antennas bent along the $E$ - (length of the patch) and $H$-planes (width of the patch) are plotted and compared in Fig. 3. It is observed that bending the antenna along the $E$ and $H$-planes increases the antenna bandwidth to $\sim 47 \%$ and $\sim 50 \%$, respectively. Since the $H$-plane bending affects the impedance bandwidth more significantly than the $E$-plane bending, the effect of varying the antenna parameters is further investigated for the $H$-plane bent for this wideband antenna.

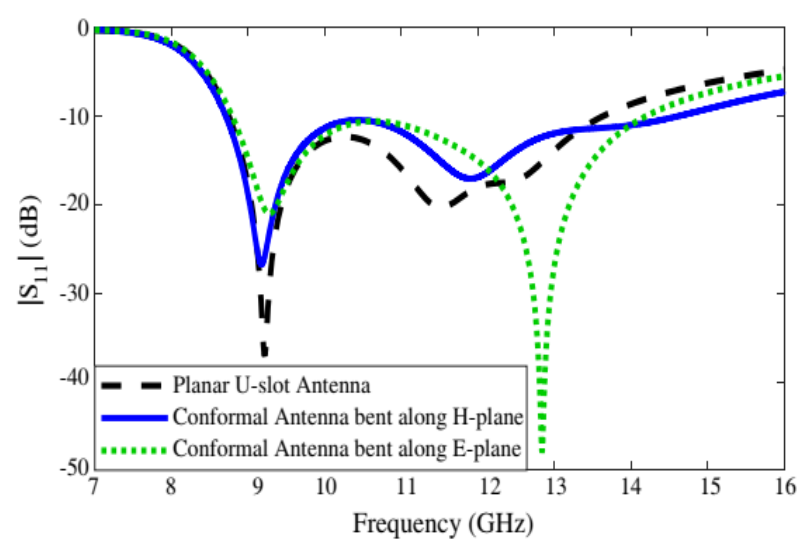

Figure 3. Comparison of the reflection coefficients of planar and conformal U-slot patch antennas bent along the $E$ - and $H$-planes, when $h=3.175 \mathrm{~mm}, \alpha=100^{\circ}$, and $r=15 \mathrm{~mm}$ [40]

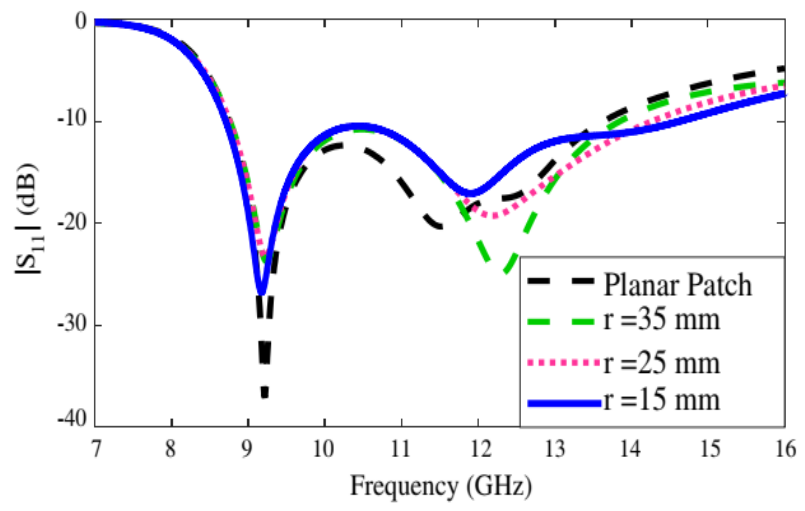

(a)

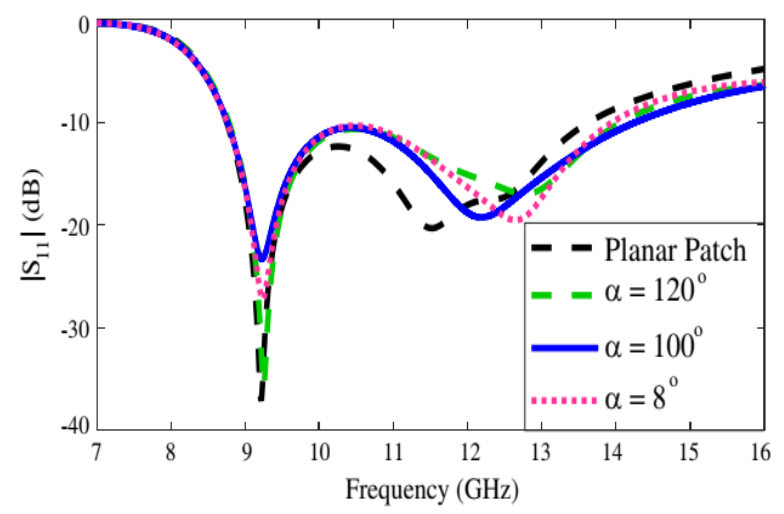

(b)

Figure 4. Reflection coefficients of the conformal U-slot antenna in Fig. 1 with (a) $r=25 \mathrm{~mm}$, as $\alpha$ varies from $80^{\circ}$ to $100^{\circ}$ and (b) $\alpha=100^{\circ}$, as $r$ changes from $15 \mathrm{~mm}$ to $35 \mathrm{~mm}$ [40]

The radius for the cylinder is maintained constant at $25 \mathrm{~mm}$, which is almost half the size of the planar earth plane and the arc angle is varied from 800 to 1,200 , to study the effect of the arc angle on the impedance bandwidth of the conformal U-slot patch antenna under investigation. Figure shows the corresponding coefficients of reflection versus frequency in fig.4 (a). It is observed that the impedance bandwidth increases to as much as $\alpha=1000$ as the arc angle changes and then decreases. This shows that the conformal U-slot antenna, with the 1000 arc angle, has $\sim 4 \%$ more bandwidth than its planar version. The conformal structure's arc angle is then fixed at 1000, reaching an impedance bandwidth of approximately $47 \%$, and the radius of the cylinder structure varies from 15 to 
$35 \mathrm{~mm}$. The results are depicted in Fig. 4(b). As can be seen, the conformal antenna now exhibits much wider impedance bandwidth in the order of $\sim 50 \%$, when the radius of curvature is equal to the $15 \mathrm{~mm}$. Thus, this conformal structure improves the bandwidth by $7 \%$ compared to its planar counterpart. This is mainly attributed to the larger volume of the conformal antenna, reducing the antenna quality factor [38], and thus widening the impedance bandwidth.

\section{CONCLUSION}

This paper analyses the various approaches and schemes that is utilized in conformal antenna arrays design. It can be concluded that, these models are based only on the convex optimization theory based synthesis. Particular conformal patch antenna can be designed for specific applications. By changing surface, polarization and pattern synthesis the presented models can be improved and yield more accurate forecasts. The pattern synthesis of conformal antenna became much fast and efficient while using optimization techniques. So, an enhancement to the design of conformal antenna along with hybrid optimization algorithm can be proposed as a future work.

\section{REFERENCES}

1. Polegre. A, G. Caille, L. Boyer and A. Roederer, "Semi-active conformal array for ESA's GAIA mission", IEEE Antennas and Propagation Society Symposium, 2004.

2. $\mathrm{Y}$. $\mathrm{Wu}$ and $\mathrm{Y}$. Cheng, "Conical Conformal Shaped-Beam Substrate-Integrated Waveguide Slot Array Antenna with Conical-to-Cylindrical Transition", IEEE Transactions on Antennas and Propagation, vol. 65, no. 8, pp. 4048-4056, 2017.

3. H. Schippers, "Radiation analysis of conformal phased array antennas on distorted structures", 2018

4. H. Schippers, J. Verpoorte, P. Jorna, A. Hulzinga, A. Meijerink, C. Roeloffzen, R. Heideman, A. Leinse and M. Wintels, "Conformal phased array with beam forming for airborne satellite communication", 2008 International ITG Workshop on Smart Antennas, 2008.

5. L. Vaskelainen, "Phase synthesis of conformal array antennas", IEEE Transactions on Antennas and Propagation, vol. 48, no. 6, pp. 987-991, 2000

6. H. Schippers, J. Verpoorte, P. Jorna, A. Hulzinga, A. Meijerink, C. Roeloffzen, R. Heideman, A. Leinse and M. Wintels, "Conformal phased array with beam forming for airborne satellite communication", 2008 International ITG Workshop on Smart Antennas, 2008.

7. L. Vaskelainen, "Phase synthesis of conformal array antennas", IEEE Transactions on Antennas and Propagation, vol. 48, no. 6, pp. 987-991, 2000.

8. P. Li, W. Xu, L. Song and Y. Qiu, "A Novel Inversion Method of Manufacturing Flaws in the Packaging of Conformal Load-Bearing Antenna Structure", 2018

9. M. Klemm and G. Troester, "Textile UWB Antennas for Wireless Body Area Networks", IEEE Transactions on Antennas and Propagation, vol. 54, no. 11, pp. 3192-3197, 2006.

10. T. Kennedy, P. Fink, A. Chu, N. Champagne, G. Lin and M. Khayat, "Body-Worn E-Textile Antennas: The Good, the Low-Mass, and the Conformal", IEEE Transactions on Antennas and Propagation, vol. 57, no. 4, pp. 910-918, 2009.

11. P. Saone, Y. Rahmat-Samii, M. Schaffrath and M. Kivikoski, "Effect of textile materials on wearable antenna performance: a case study of GPS antennas", IEEE Antennas and Propagation Society Symposium, 2004.

12. Zheyu Wang, Lanlin Zhang, Y. Bayram and J. Volakis, "Multilayer printing of embroidered RF circuits on polymer composites", 2011 IEEE International Symposium on Antennas and Propagation (APSURSI), 2011.

13. Y. Bayram, Y. Zhou, B. Shim, S. Xu, J. Zhu, N. Kotov and J. Volakis, "E-Textile Conductors and Polymer Composites for Conformal Lightweight Antennas", IEEE Transactions on Antennas and Propagation, vol. 58, no. 8, pp. 2732-2736, 2010.
14. S. Morris, Y. Bayram, L. Zhang, Z. Wang, M. Shtein and J. Volakis, "High-Strength, Metalized Fibers for Conformal Load Bearing Antenna Applications", IEEE Transactions on Antennas and Propagation, vol. 59, no. 9, pp. 3458-3462, 2011.

15. K. Wincza and S. Gruszczynski, "Influence of Curvature Radius on Radiation Patterns in Multibeam Conformal Antennas", 2006 European Microwave Conference, 2006.

16. G. Oliveri, P. Rocca and A. Massa, "Differential evolution as applied to electromagnetics: Advances, comparisons, and applications", 2012 6th European Conference on Antennas and Propagation (EUCAP), 2012.

17. B. Xu, "Robust adaptive neural control of flexible hypersonic flight vehicle with dead-zone input nonlinearity", Nonlinear Dynamics, vol. 80, no. 3, pp. 1509-1520, 2015.

18. J. Helander, K. Zhao, Z. Ying and D. Sjoberg, "Performance Analysis of Millimeter-Wave Phased Array Antennas in Cellular Handsets", IEEE Antennas and Wireless Propagation Letters, vol. 15, pp. 504-507, 2016

19. B. Braaten, S. Roy, S. Nariyal, M. Al Aziz, N. Chamberlain, I. Irfanullah, M. Reich and D. Anagnostou, "A Self-Adapting Flexible (SELFLEX) Antenna Array for Changing Conformal Surface Applications", IEEE Transactions on Antennas and Propagation, vol. 61, no. 2, pp. 655-665, 2013.

20. Y. Cheng, H. Xu, D. Ma, J. Wu, L. Wang and Y. Fan, "Millimeter-Wave Shaped-Beam Substrate Integrated Conformal Array Antenna", IEEE Transactions on Antennas and Propagation, vol. 61, no. 9, pp. 4558-4566, 2013.

21. B. Braaten, S. Roy, I. Irfanullah, S. Nariyal and D. Anagnostou, "Phase-Compensated Conformal Antennas for Changing Spherical Surfaces", IEEE Transactions on Antennas and Propagation, vol. 62 , no. 4, pp. $1880-1887,2014$

22. M. Khan, B. Ijaz, D. Anagnostou, B. Braaten, A. Capobianco and S. Asif, "Frequency reconfigurable self-adapting conformal array for changing surfaces", IET Microwaves, Antennas \& Propagation, vol. 10, no. 8, pp. 897-901, 2016.

23. Y. Zhao, Z. Shen and W. Wu, "Conformal SIW H-Plane Horn Antenna on a Conducting Cylinder", IEEE Antennas and Wireless Propagation Letters, vol. 14, pp. 1271-1274, 2015

24. X. Lan, L. Wang, Y. Wang, C. Choi and D. Choi, "Tensor 2-D DOA Estimation for a Cylindrical Conformal Antenna Array in a Massive MIMO System Under Unknown Mutual Coupling", IEEE Access, vol. 6, pp. 7864-7871, 2018.

25. L. Wan, G. Han, J. Jiang, C. Zhu and L. Shu, "A DOA Estimation Approach for Transmission Performance Guarantee in D2D Communication", Mobile Networks and Applications, vol. 22, no. 6, pp. 998-1009, 2017.

26. L. Wan, W. Si, L. Liu, Z. Tian and N. Feng, "High Accuracy 2D-DOA Estimation for Conformal Array Using PARAFAC", International Journal of Antennas and Propagation, vol. 2014, pp. $1-14,2014$

27. R. Li, L. Xu, X. Shi, L. Chen and C. Cui, "Two-Dimensional NC-Music DOA Estimation Algorithm with a Conformal Cylindrical Antenna Array", Journal of Electromagnetic Waves and Applications, vol. 25, no. 5-6, pp. 805-818, 2011.

28. Y. Zou, H. Xie, L. Wan, G. Han and W. Li, "2D-DOA and Mutual Coupling Estimation in Vehicle Communication System via Conformal Array", Mobile Information Systems, vol. 2015, pp. 1-10, 2015.

29. Z. Liang, J. Ouyang and F. Yang, "A hybrid GA-PSO optimization algorithm for conformal antenna array pattern synthesis", Journal of Electromagnetic Waves and Applications, vol. 32, no. 13, pp. 1601-1615, 2018.

30. M. Comisso and R. Vescovo, "Fast 3D Pattern Synthesis with Polarization and Dynamic Range Ratio Control for Conformal Antenna Arrays", International Journal of Antennas and Propagation, vol. 2014, pp. 1-9, 2014.

31. R. Karimzadeh Baee, K. Forooraghi and S. Chamaani, "Conformal Array Pattern Synthesis Using a Hybrid WARP/2LB-MOPSO Algorithm", International Journal of Antennas and Propagation, vol. 2012, pp. 1-7, 2012

32. M. Comisso and R. Vescovo, "Fast Co-Polar and Cross-Polar 3D Pattern Synthesis With Dynamic Range Ratio Reduction for Conformal Antenna Arrays", IEEE Transactions on Antennas and Propagation, vol. 61, no. 2, pp. 614-626, 2013.

33. K. Yang, Z. Zhao, J. Ouyang, Z. Nie and Q. Liu, "Optimization method on conformal array element positions for low side lobe pattern synthesis", IET Microwaves, Antennas 
\& Propagation, vol. 6, no. 6, p. 646, 2012.

34. J. Ouyang, J. Zhang, K. Zhi Zhang, J. Yang, X. Luo and F. Yang, "Analysis and synthesis of conformal conical surface linear phased array with volume surface integral equation+AEP (Active Element Pattern) and INSGA-II", IET Microwaves, Antennas \& Propagation, vol. 6, no. 11, pp. 1277-1285, 2012.

35. Y. Bai, S. Xiao, C. Liu and B. Wang, "A Hybrid IWO/PSO Algorithm for Pattern Synthesis of Conformal Phased Arrays", IEEE Transactions on Antennas and Propagation, vol. 61, no. 4, pp. 2328-2332, 2013.

36. Z. Huang, H. Zhang and J. Zhou, "Full polarimetric sum and difference patterns synthesis for conformal array", Electronics Letters, vol. 51, no. 8, pp. 602-604, 2015.

37. W. Hu, X. Wang, Y. Li and S. Xiao, "Synthesis of Conformal Arrays With Matched Dual-Polarized Patterns", IEEE Antennas and Wireless Propagation Letters, vol. 15, pp. 1341-1344, 2016.

38. Luk, K., K.-F. Lee, and J.S. Dahele, "Analysis of the cylindrical rectangular patch antenna," IEEE Trans. Antennas Propag., Vol. 37, No. 2, 143-147, Feb. 1989

39. "ANSYS high frequency electromagnetic field simulator HFSS (version 18.1)," Canonsburg, PA

40. Tanzeela Mitha and Maria Pour, "Conformal Wideband Microstrip Patch Antennas on Cylindrical Platforms", Progress In Electromagnetics Research Letters, Vol. 80, 1-6, 2018.

Published By: 